EUROPEAN ORGANIZATION FOR NUCLEAR RESEARCH

CERN-EP/2002-046

20 June 2002

\title{
Detector Physics and Simulation of Resistive Plate Chambers
}

\author{
W. Riegler ${ }^{1)}$, C. Lippmann ${ }^{1)}$, R. Veenhof ${ }^{1)}$
}

\begin{abstract}
We present a simulation model suited to study efficiency, timing and pulse-height spectra of Resistive Plate Chambers. After discussing the details of primary ionisation, avalanche multiplication, signal induction and frontend electronics, we apply the model to timing RPCs with time resolutions down to $50 \mathrm{ps}$ and trigger RPCs with time resolutions of about $1 \mathrm{~ns}$.
\end{abstract}

Submitted to Nuclear Instruments and Methods

1) EP Division, CERN, CH-1211 Geneva 23, Switzerland 


\section{Introduction}

A detailed study of signal induction and signal propagation in RPCs can be found in [1] and [2]. In this report we focus on the detector physics of RPCs, especially the primary ionisation and avalanche statistics. We present analytical formulae for average signals, charges, time resolution and efficiency to study the 'order of magnitude' behaviour of RPCs. We describe a simple Monte Carlo procedure that enables us to simulate accurately the detector physics processes. Effects of high fields, like the change in avalanche statistics and space charge effects, are also discussed and analysed. Simulation of charge spectra and efficiency of RPCs was reported in several papers [3][4][5]. In addition to these characteristics, we want to study signal development, time resolution as well as frontend electronics and noise effects. As starting values for the gas properties we use the numbers predicted by Heed [6], Magboltz [7] and Imonte [8].

To illustrate the simulation procedure we will show the results for two different kinds of devices. First we will investigate timing RPCs with a $300 \mu \mathrm{m}$ gap, similar to the ones developed by P. Fonte et al. [9][10][11] with time resolutions down to $50 \mathrm{ps}$ (Fig. 1). Similar geometries with $250 \mu \mathrm{m}$ gap are described in [12]. Then we will study $2 \mathrm{~mm}$ gap RPCs similar to the ones in ATLAS [13][14] with a time resolution of $\approx 1 \mathrm{~ns}$, used for triggering the experiment (Fig. 2). Both RPC types operate in saturated avalanche mode with a gas mixture of $\mathrm{C}_{2} \mathrm{~F}_{4} \mathrm{H}_{2} / \mathrm{i}-\mathrm{C}_{4} \mathrm{H}_{10} / \mathrm{SF}_{6}$ [15].

The timing RPCs by P. Fonte et al. use gas gaps of $300 \mu \mathrm{m}$ and resistive glass plates with a volume resistivity of about $2 \times 10^{12} \Omega \mathrm{cm}$. The gas is $\mathrm{C}_{2} \mathrm{~F}_{4} \mathrm{H}_{2} / \mathrm{i}-\mathrm{C}_{4} \mathrm{H}_{10} / \mathrm{SF}_{6} 85 / 5 / 10$ at an operating voltage of $6(3) \mathrm{kV}$ for the double(single) gap RPCs resulting in an electric field of about $100 \mathrm{kV} / \mathrm{cm}$ in the gas gaps. The ATLAS RPCs use $2 \mathrm{~mm}$ Bakelite with a volume resistivity of $9 \times 10^{9} \Omega \mathrm{cm}$. The $2 \mathrm{~mm}$ gas gap is filled with $\mathrm{C}_{2} \mathrm{~F}_{4} \mathrm{H}_{2} / \mathrm{i}-\mathrm{C}_{4} \mathrm{H}_{10} / \mathrm{SF}_{6} 97 / 2.5 / 0.5$. The working point is around $10 \mathrm{kV}$ giving an electric field of $50 \mathrm{kV} / \mathrm{cm}$ in the gas gap.

a)
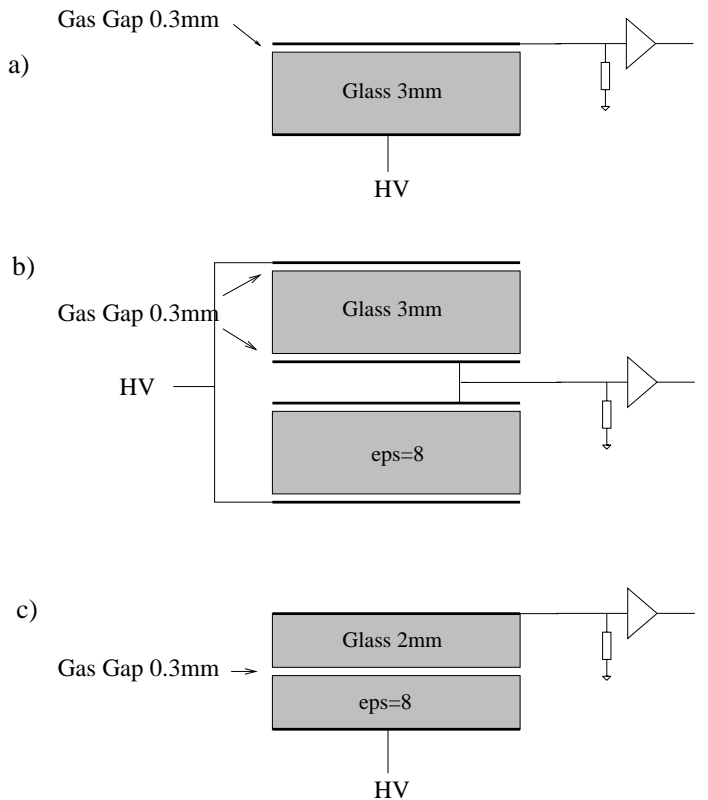

Figure 1: RPC geometries similar to the ones developed by P. Fonte et al. [9][10][11]. 


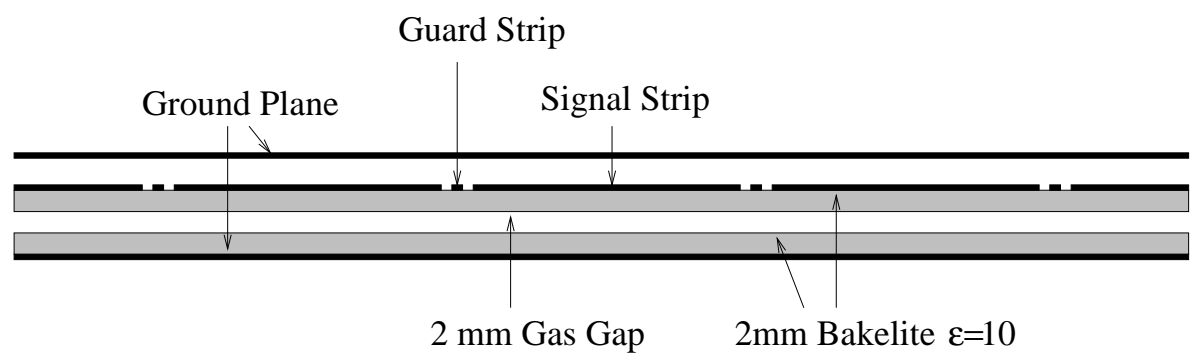

Figure 2: RPC geometry similar to the one used for ATLAS [14]. 
Primary Ionisation

The charge deposit is characterised by the average number of clusters per unit of length and the probability distribution for the number of electrons per cluster. The numbers are calculated using Heed [6]. The average number of clusters/mm versus $(\gamma-1)$ of the particle is shown in Fig. 3a). For the RPC gas we find an average of 7.5 clusters $/ \mathrm{mm}$ for a minimum ionising particle. The predicted numbers of isobutane and methane are shown as a reference since measurements for the gases are available [16]. The prediction from Heed matches the experimental results quite well, it should however be mentioned that the experimental numbers vary significantly in the literature. For a $10 \mathrm{GeV}$ pion we find on average 9.5 clusters $/ \mathrm{mm}$, so the average distance between clusters is $\lambda=105 \mu \mathrm{m}$. The cluster size distribution for two gases is shown in Fig. 3b. The distance between the clusters is exponentially distributed, so the proba-

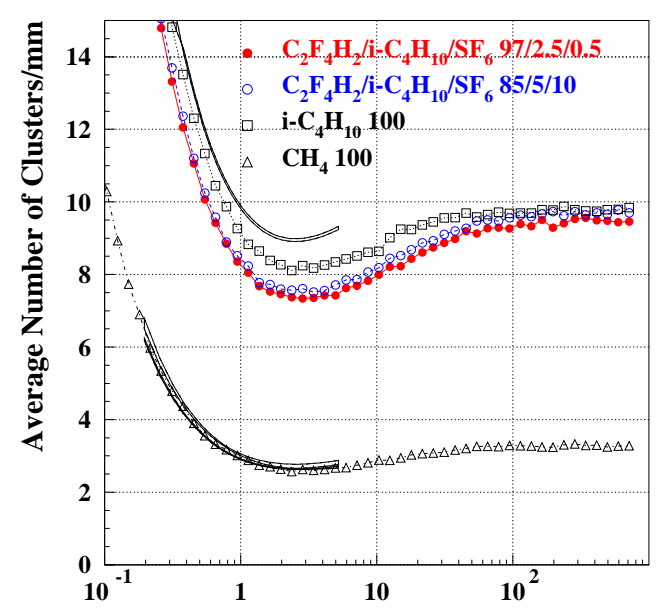

a)

$(\gamma-1)$

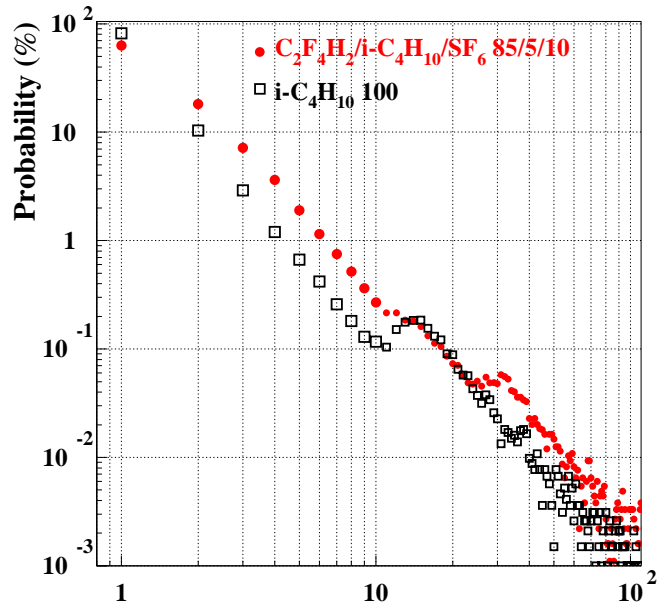

Electrons per Cluster

b)

Figure 3: (a) Average number of clusters/mm for different gases predicted by Heed [6]. The 'solid bands' show measurements for methane and isobutane from [16]. (b) Cluster size distribution for a pion energy of $10 \mathrm{GeV}$ as simulated by Heed. Cutting at 500 electrons the average number of electrons/cluster is 2.45 for the RPC gas.

bility to find the first cluster between position $x$ and $x+\mathrm{d} x$ is

$$
P(x)=\frac{1}{\lambda} e^{-\frac{x}{\lambda}}
$$

The probability for the $n^{\text {th }}$ cluster to be between position $x$ and $x+\mathrm{d} x$, independent of the position of all the others, is given by

$$
\begin{aligned}
P_{c l u}(n, x) & =\int_{0}^{x} \int_{0}^{x_{n-1}} \ldots \int_{0}^{x_{2}} P\left(x_{1}\right) P\left(x_{2}-x_{1}\right) \ldots P\left(x-x_{n-1}\right) \mathrm{d} x_{1} \mathrm{~d} x_{2} \ldots \mathrm{d} x_{n-1} \\
& =\frac{x^{n-1}}{(n-1) ! \lambda^{n}} e^{-\frac{x}{\lambda}} \quad(\Gamma \text { distribution })
\end{aligned}
$$

with an average distance from the gas gap edge of $\bar{x}=n \lambda$. For the simulation we simply put the primary clusters with distances according to Eq. 1 and the number of electrons for each cluster from the cluster size distribution in Fig. 3 b. 


\section{Secondary particles}

Secondary particles created by the incident particle in the RPC material potentially have a very big impact on the RPC performance since these particles, mostly delta electrons, create many ionisation electrons at the 'beginning' of the gas gap. For the RPCs in Fig. 1a) and b) the particle enters the gas gap through an aluminium plate. A calculation with Fluka [17] [18] for a $7 \mathrm{GeV}$ pion crossing a $3 \mathrm{~mm}$ aluminium plate shows that the probability that the pion is accompanied by at least one charge particle is only $4.92 \%$. Therefore the secondaries should not have a serious influence on the charge spectrum, efficiency and timing.

\section{$4 \quad$ Avalanche Multiplication}

Each electron will start an avalanche which will grow until it hits the resistive plate or metal electrode. Avalanche multiplication for electro-negative gases at high fields is described in detail in [19]. In case the probability that an electron multiplies is independent of the previous position of multiplication, the avalanche development is characterised by the Townsend coefficient $\alpha$ and attachment coefficient $\eta$. Fig. 4 shows these parameters as calculated with Imonte [8]. For the trigger RPCs with $E=50 \mathrm{kV} / \mathrm{cm}$ we expect an effective Townsend coefficient of around 10/mm while for the timing RPCs with $E=100 \mathrm{kV} / \mathrm{cm}$ we expect a value around $100 / \mathrm{mm}$. If the avalanche contains $n$ electrons at position $x$ the probability that it contains $n+1$

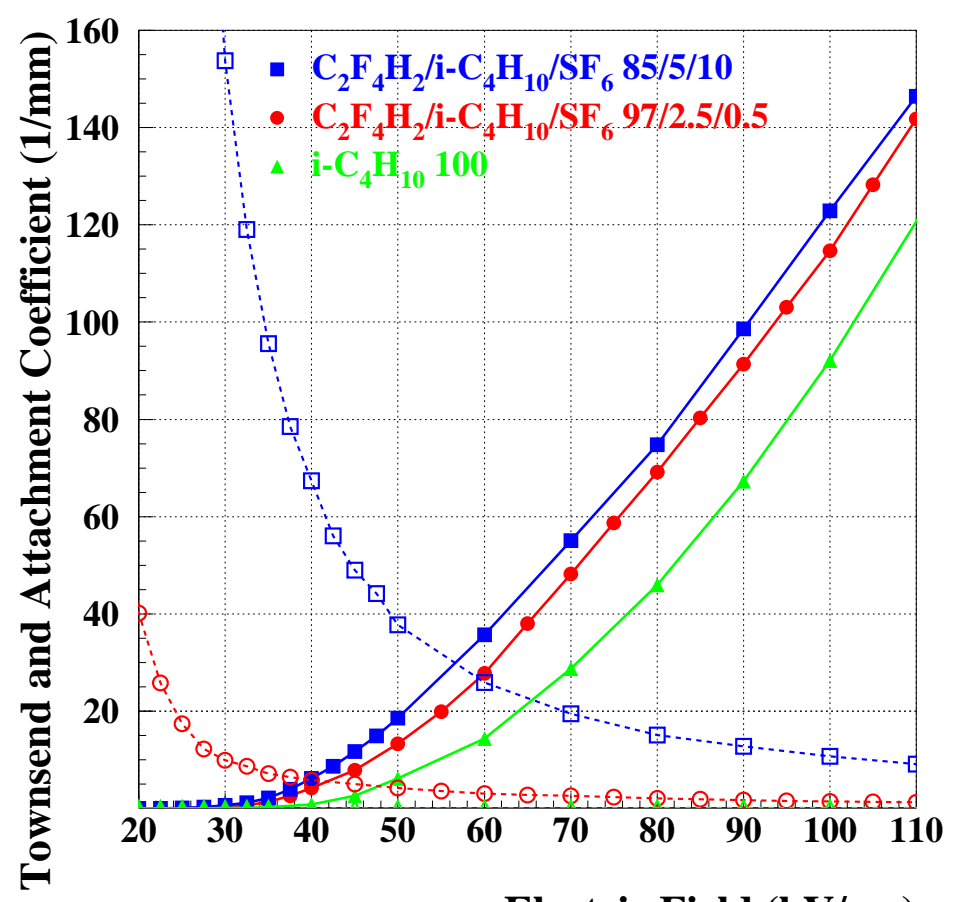

Electric Field (kV/cm)

Figure 4: Townsend and attachment coefficient as calculated by IMONTE [8].

at $x+\mathrm{d} x$ is given by $n \alpha \mathrm{d} x$. Following the same arguments the probability that for an avalanche of size $n$, one electron gets attached (forming a negative ion) over distance $\mathrm{d} x$ is $n \eta \mathrm{d} x$. For the average number of electrons $\bar{n}$ and positive ions $\bar{p}$ we therefore have the relations

$$
\frac{\mathrm{d} \bar{n}}{\mathrm{~d} x}=(\alpha-\eta) \bar{n} \quad \frac{\mathrm{d} \bar{p}}{\mathrm{~d} x}=\alpha \bar{n}
$$


with $\bar{n}(0)=1$ and $\bar{p}(0)=0$ giving the solution

$$
\bar{n}(x)=e^{(\alpha-\eta) x} \quad \bar{p}(x)=\frac{\alpha}{\alpha-\eta}\left(e^{(\alpha-\eta) x}-1\right)
$$

The average number of negative ions is then $\bar{p}-\bar{n}$. To derive the statistical fluctuation of the avalanche, we proceed as shown in [19]. The probability $P(n, x)$ for an avalanche started with a single electron to contain $n$ electrons after distance $x$ is defined by

$$
\begin{array}{rlr}
P(n, x+\mathrm{d} x)= & P(n-1, x)(n-1) \alpha \mathrm{d} x(1-(n-1) \eta \mathrm{d} x)+ \\
& P(n, x)(1-n \alpha \mathrm{d} x)(1-n \eta \mathrm{d} x) & + \\
& P(n, x) n \alpha \mathrm{d} x n \eta \mathrm{d} x & + \\
& P(n+1, x)(1-(n+1) \alpha \mathrm{d} x)(n+1) \eta \mathrm{d} x
\end{array}
$$

The four lines represent the four possibilities to find $n$ electrons at position $x+\mathrm{d} x$. The first line gives the probability that there are $n-1$ electrons at $x$, exactly one of them duplicates and no electron is attached. The second line gives the probability that there are $n$ electrons at $x$, no electron duplicates and no electron is attached. The third line gives the probability that from $n$ electrons, one multiplies and one gets attached and finally the fourth line gives the probability that from $n+1$ electrons one gets attached and no electron is multiplied. Evaluating the expression and omitting the higher order terms of $\mathrm{d} x$ we find

$$
\frac{\mathrm{d} P(n, x)}{\mathrm{d} x}=-P(n, x) n(\alpha+\eta)+P(n-1, x)(n-1) \alpha+P(n+1, x)(n+1) \eta
$$

with the general solution

$$
\begin{aligned}
P(n, x) & =k \frac{\bar{n}(x)-1}{\bar{n}(x)-k} & & n=0 \\
& =\bar{n}(x)\left(\frac{1-k}{\bar{n}(x)-k}\right)^{2}\left(\frac{\bar{n}(x)-1}{\bar{n}(x)-k}\right)^{n-1} & & n>0
\end{aligned}
$$

where

$$
\bar{n}(x)=e^{(\alpha-\eta) x} \quad k=\frac{\eta}{\alpha}
$$

The variance $\sigma^{2}(x)$ of the distribution is given by

$$
\sigma^{2}(x)=\left(\frac{1+k}{1-k}\right) \bar{n}(x)(\bar{n}(x)-1)
$$

We see that the average electron number depends on the so called effective Townsend coefficient $\alpha_{\text {eff }}=\alpha-\eta$, the variance and the distribution itself however depend also on $k=\eta / \alpha$ explicitly. For illustration, Fig. 5 shows the above distribution for the same effective Townsend coefficient but different $\alpha$ and $\eta$. For a distance $x$ where $\bar{n}$ is sufficiently large, we can approximate the above formula and find

$$
\begin{aligned}
P(n, x) & =k & & n=0 \\
& =\frac{(1-k)^{2}}{\bar{n}(x)} \exp \left[-(1-k) \frac{n}{\bar{n}(x)}\right] & & n>0
\end{aligned}
$$




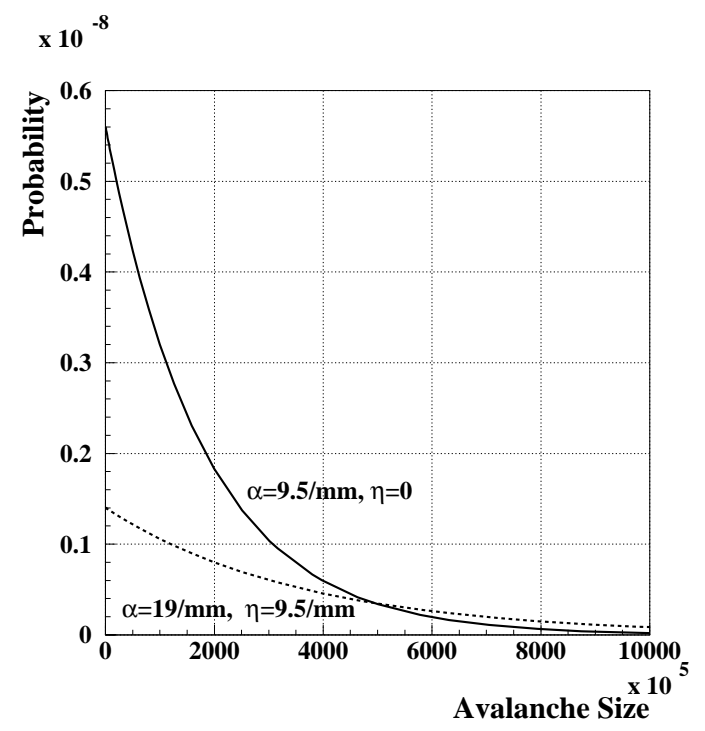

Figure 5: Charge distribution for avalanches starting with a single electron. The effective Townsend coefficient $\alpha-\eta$ is the same for both curves.

In the case that $\alpha=\eta$ or $\alpha=0$ the distribution from Eq. 7 becomes undefined and we have to use different expressions. In case $\alpha=\eta$ the probabilities are

$$
\begin{aligned}
P(n, x) & =\frac{\alpha x}{1+\alpha x} & n & =0 \\
& =\frac{1}{(1+\alpha x)^{2}}\left(\frac{\alpha x}{1+\alpha x}\right)^{n-1} & & n>0
\end{aligned}
$$

and the variance becomes

$$
\sigma^{2}(x)=2 \alpha x
$$

In case $\alpha=0$ the probabilities are

$$
\begin{aligned}
P(n, x) & =1-e^{-\eta x} & & n=0 \\
& =e^{-\eta x} & & n=1
\end{aligned}
$$

and the probability to find $n>1$ electrons is zero. The variance is

$$
\sigma^{2}(x)=e^{-2 \eta x}\left(e^{\eta x}-1\right)
$$

To generate a random number according to Eq. 7 one draws a uniform random number $s$ from the interval $(0,1)$ and calculates

$$
\begin{array}{rlr}
n & =0 & s<k \frac{\bar{n}(x)-1}{\bar{n}(x)-k} \\
& =1+\text { Trunc }\left[\frac{1}{\ln \left(1-\frac{1-k}{\bar{n}(x)-k}\right)} \ln \left(\frac{(\bar{n}(x)-k)(1-s)}{\bar{n}(x)(1-k)}\right)\right] & s>k \frac{\bar{n}(x)-1}{\bar{n}(x)-k}
\end{array}
$$

where 'Trunc' means truncation of the decimals. In case $\bar{n}(x)$ is very large the numerical evaluation of the first factor can become problematic and it is better to use the series expansion for $\ln (1-x)=-\left(x+\frac{1}{2} x^{2}+\frac{1}{3} x^{3}+\ldots\right)$. 
To generate a random number according to Eq. 11 one draws a uniform random number $s$ from the interval $(0,1)$ and calculates

$$
\begin{array}{rlrl}
n & =0 & & s<\frac{\alpha x}{1+\alpha x} \\
& =1+\text { Trunc }\left[\frac{1}{\ln \left(\frac{\alpha x}{1+\alpha x}\right)} \ln ((1-s)(1+\alpha x))\right] & s>\frac{\alpha x}{1+\alpha x}
\end{array}
$$

To generate a random number according to Eq. 13 one calculates

$$
\begin{array}{ll}
n=0 & s>e^{-\eta x} \\
n=1 & s<e^{-\eta x}
\end{array}
$$

If we want to calculate the induced signal, we have to simulate the avalanche development instead of using the probability distribution for the final avalanche charge. Let's first follow the avalanche development for a single initial electron starting at one edge of the gas gap. We divide the gap into $N$ steps of $\Delta x$. The average multiplication $\bar{n}(\Delta x)$ for a single electron over this distance is given by $e^{(\alpha-\eta) \Delta x}$. Starting with one electron at $x=0$ we find $n_{1}$ electrons at $x=\Delta x$ where $n_{1}$ is from Eq. $15,16,17$. Each of these electrons will again multiply the same way. To find the number $n_{2}$ of electrons at $x=2 \Delta x$ we loop over the $n_{1}$ electrons, draw a number from Eq. 15, 16, 17 for each electron and sum them. This procedure can be repeated through the full gap, but it is very time consuming. If the number of electrons $n_{i}$ at a given distance of $i \Delta x$ is sufficiently large we can use the central limit theorem and calculate the number of electrons $n_{i+1}$ at distance $(i+1) \Delta x$ by drawing a random number from a Gaussian with mean $\mu$ and sigma $\sigma_{\mu}$ of

$$
\mu=n_{i} \bar{n}(\Delta x) \quad \sigma_{\mu}=\sqrt{n_{i}} \sigma(\Delta x)
$$

where $\sigma(x)$ is from Eq. 9, 12, 14. This makes the simulation procedure very fast. Fig. 6 shows examples of individual avalanches starting from a single electron. The very beginning of the avalanche decides on the final avalanche size. Once the number of electrons has reached a certain size the avalanche grows smoothly like $e^{(\alpha-\eta) x}$. 


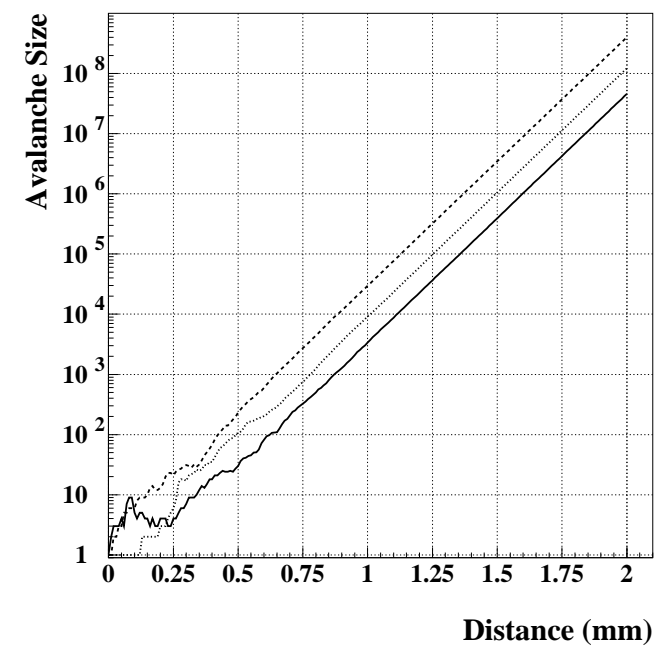

Figure 6: Avalanches started by a single electron at $x=0$ for $\alpha=13 / \mathrm{mm}, \eta=3.5 / \mathrm{mm}$. We see that the very beginning of the avalanche decides on the final avalanche size. Once the number of electrons is sufficiently large the avalanche grows like $e^{(\alpha-\eta) x}$. 
The movement of the electrons in the electric field finally induces a current signal on the RPC electrodes. The negative and positive ions induce a signal which is much smaller due to their slow drift velocity which we will neglect in the following. Fig. 7 shows the drift velocities for different gases as predicted by Magboltz [7]. The current signal induced on an electrode is

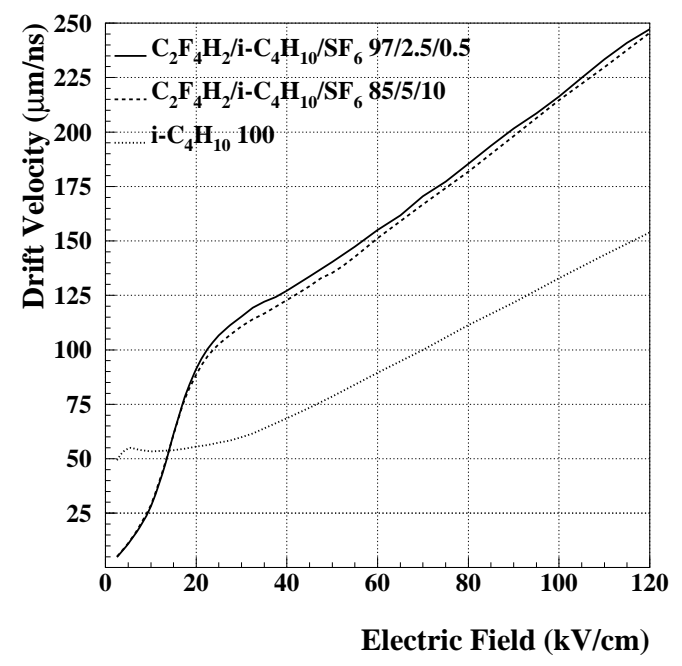

Figure 7: Drift velocity for different gases as predicted by Magboltz [7].

given by [20]

$$
i(t)=\frac{E_{w} \cdot v}{V_{w}} e_{0} N(t)
$$

where $e_{0}$ is the electron charge, $E_{w}$ (weighting field) is the electric field in the gas gap if we put the electrode to potential $V_{w}$ and ground all other electrodes, $v$ is the electron drift velocity and $N(t)$ is the number of electrons present at time $t$ which we calculate by simulating the avalanches of the individual primary electrons. The weighting fields $E_{w} / V_{w}$ for the geometries in Fig. 1 and 2 (considering the electrodes to be large compared to the RPC thickness) are given by
1a) $\frac{E_{w}}{V_{w}}=\frac{\epsilon_{r}}{b+d \epsilon_{r}}$
1b) $\frac{E_{w}}{V_{w}}=\frac{\epsilon_{r}}{b+2 d \epsilon_{r}}$
1c) 2) $\frac{E_{w}}{V_{w}}=\frac{\epsilon_{r}}{2 b+d \epsilon_{r}}$

where $\epsilon_{r}$ is the Bakelite (glass) permittivity, $b$ the Bakelite (glass) thickness and $d$ the gas gap. To get an idea about the signals we first assume a single primary electron somewhere in the gas gap. Using the result that after some initial fluctuations $N(t)$ grows like a smooth exponential (Fig. 6) and that the charge after a fixed distance (time) is exponentially distributed (Eq. 10), we can assume an RPC signal distribution of

$$
i(t)=A e^{(\alpha-\eta) v t} \quad P(A)=\frac{1}{A_{a v}} e^{-\frac{A}{A_{a v}}}
$$

where $P(A)$ is the probability to find the amplitude $A$ in an event. This signal growth distribution is independent of the position of the primary electron in the gas gap. The position only determines when the avalanche hits the electrode i.e. it determines when the signal is stopped. 
If the gas gap is large compared to the average distance between clusters, the signal is formed by many clusters. To get an idea of the average pulse height and signal shape, we assume $N_{0}=d / \lambda$ clusters distributed evenly in the gas gap, each containing $n_{a v}$ electrons ( $d$ is the gap thickness and $\lambda$ is the average distance between clusters). The signal is then given by

$$
I(t)=\frac{E_{w}}{V_{w}} e_{0} v N(t) \quad N(t)=\sum_{n=1}^{N_{0}} n_{a v} e^{(\alpha-\eta) v t} \Theta\left[\frac{d}{v}\left(1-\frac{n}{N_{0}}\right)-t\right]
$$

where $\Theta(x)$ is the step function. The enveloping function of this signal is

$$
I_{e n v}(t)=\frac{E_{w}}{V_{w}} e_{0} v N_{e n v}(t) \quad N_{e n v}(t)=N_{0} n_{a v}\left(1-\frac{t v}{d}\right) e^{(\alpha-\eta) v t} \Theta\left(\frac{d}{v}-t\right)
$$

Both of these functions are shown in Fig. 8. These formulas only match to reality if saturation effects can be neglected.

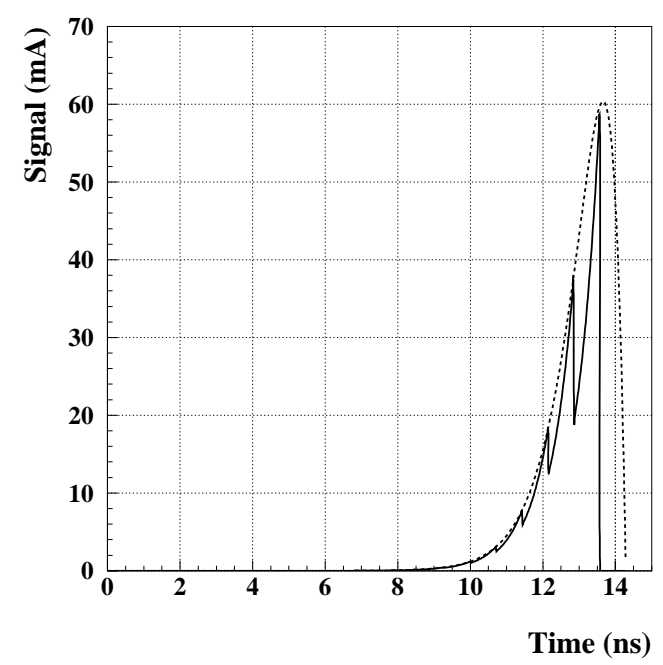

Figure 8: Average signal from Eq. (22) with the enveloping function from Eq. (23) for the Trigger RPC geometry with parameters from Eq. 25.

To discuss the performance numbers in the next sections we use the parameters from the previous figures at typical operating voltages:

$$
\begin{array}{cccc}
\text { Timing RPC: } & E=100 \mathrm{kV} / \mathrm{cm} \quad \alpha=123 / \mathrm{mm} \quad \eta=10.5 / \mathrm{mm} \\
v=215 \mu \mathrm{m} / \mathrm{ns} \quad d=0.3 \mathrm{~mm} \quad \varepsilon_{r}=8 \\
\lambda=0.1 \mathrm{~mm} \quad n_{a v}=2.45 \quad b=2 \mathrm{~mm} \\
\text { Trigger RPC: } \quad E=50 \mathrm{kV} / \mathrm{cm} \quad \alpha=13.3 / \mathrm{mm} \quad \eta=4.2 \times 0.45 / \mathrm{mm} \\
v=140 \mu \mathrm{m} / \mathrm{ns} \quad d=2 \mathrm{~mm} \quad \varepsilon_{r}=10 \\
\lambda=0.1 \mathrm{~mm} \quad n_{a v}=2.45 \quad b=2 \mathrm{~mm}
\end{array}
$$

where $\alpha$ is the Townsend coefficient, $\eta$ the attachment coefficient, $d$ the gas gap, $\epsilon_{r}$ the Bakelite (glass) permittivity, $\lambda$ the average distance between clusters, $n_{a v}$ the average number of electrons per cluster, $b$ the Bakelite (glass) thickness and $v$ the electron drift velocity. The scaling factor 0.45 for the attachment coefficient for the trigger RPC is applied to match the simulation with measurements (as shown later). 
In the following we will derive analytic expressions for the average signal and charge produced by the individual clusters as well as the total charge deposit. Comparing these formulas to measurements will show the importance of saturation effects in RPCs. We will frequently use the integral

$$
\int_{0}^{R} x^{n} e^{-x} d x=n !(1-K[R, n+1]) \quad \text { with } \quad K[R, n]=e^{-R} \sum_{k=0}^{n-1} \frac{R^{k}}{k !}
$$

\subsection{Individual Clusters}

Using $P_{\mathrm{clu}}$ from Eq. 2, the average number of electrons $\bar{N}_{n}^{-}$produced by the $n^{\text {th }}$ cluster is given by

$$
\begin{aligned}
\bar{N}_{n}^{-} & =\int_{0}^{d} P_{\mathrm{clu}}(n, x) n_{a v} \bar{n}(d-x) \mathrm{d} x \\
& =\frac{n_{a v} e^{(\alpha-\eta) d}}{[1+(\alpha-\eta) \lambda]^{n}}\left(1-K\left[d\left(\alpha-\eta+\frac{1}{\lambda}\right), n\right]\right) \\
& \approx \frac{n_{a v} e^{(\alpha-\eta) d}}{[1+(\alpha-\eta) \lambda]^{n}} \quad \text { for } \quad e^{(\alpha-\eta) d}>>1
\end{aligned}
$$

The average number of positive ions $\bar{N}_{n}^{+}$produced by the $n^{\text {th }}$ cluster is

$$
\begin{aligned}
\bar{N}_{n}^{+}= & \int_{0}^{d} P_{c l u}(n, x) n_{a v} \bar{p}(d-x) \mathrm{d} x \\
= & \frac{n_{a v} \alpha}{\alpha-\eta}\left[\frac{e^{(\alpha-\eta) d}}{[1+(\alpha-\eta) \lambda]^{n}}\left(1-K\left[d\left(\alpha-\eta+\frac{1}{\lambda}\right), n\right]\right)-\left(1-K\left[\frac{d}{\lambda}, n\right]\right)\right] \\
& \approx \frac{\alpha n_{a v} e^{(\alpha-\eta) d}}{(\alpha-\eta)[1+(\alpha-\eta) \lambda]^{n}} \quad \text { for } \quad e^{(\alpha-\eta) d}>>1
\end{aligned}
$$

The average signal from a single electron starting at position $x$ in the RPC gap is

$$
i(t, x)=\frac{E_{w}}{V_{w}} e_{0} v e^{(\alpha-\eta) v t} \Theta\left(\frac{d-x}{v}-t\right)
$$

and the corresponding induced charge is

$$
Q^{i n d}(d-x)=\int_{0}^{\infty} i(t, x) \mathrm{d} t=\frac{E_{w} e_{0}}{V_{w}(\alpha-\eta)}\left(e^{(\alpha-\eta)(d-x)}-1\right)=\frac{E_{w}}{V_{w}} \frac{1}{\alpha} \bar{p}(d-x)
$$

Therefore the average charge $\bar{Q}_{n}^{\text {ind }}$ induced by the $n^{\text {th }}$ cluster is

$$
\bar{Q}_{n}^{i n d}=\int_{0}^{d} P_{c l u}(n, x) n_{a v} Q_{n}^{i n d}(d-x) \mathrm{d} x=\frac{E_{w} e_{0}}{V_{w}} \frac{1}{\alpha} \bar{N}_{n}^{+}
$$

and hence the ratio of induced charge and ion charge, which is equal to the total avalanche charge, measures the Townsend coefficient independent of attachment:

$$
\frac{\bar{Q}_{n}^{\text {ind }}}{\bar{Q}_{n}^{\text {avalanche }}}=\frac{E_{w}}{V_{w}} \frac{1}{\alpha}
$$

The average signal due to the $n^{\text {th }}$ cluster is given by

$$
\begin{aligned}
\bar{i}_{n}(t) & =\int_{0}^{d} P_{c l u}(n, x) n_{a v} i(t, x) \mathrm{d} x \\
& =\frac{E_{w}}{V_{w}} e_{0} v n_{a v} e^{(\alpha-\eta) t}\left(1-K\left[\frac{1}{\lambda}(d-v t), n\right]\right) \Theta\left(\frac{d}{v}-t\right)
\end{aligned}
$$

The average signals of the first four clusters for the parameters from Eq. 25 are shown in Fig. 9. 


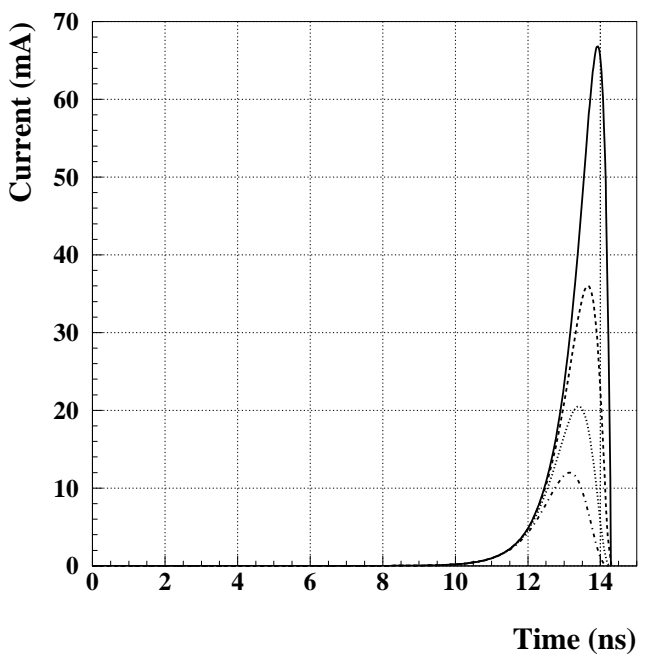

Figure 9: Average signals from the first four clusters for the parameters from Eq. 25.

\subsection{All Clusters}

In this section we calculate the average total charge and signal. The distance between the individual clusters is exponentially distributed

$$
P(\Delta x)=\frac{1}{\lambda} e^{-\frac{\Delta x}{\lambda}}
$$

Therefore the probability to have the first cluster at position $x_{1}$, the second one at position $x_{2}>x_{1}, \ldots$, the $n^{\text {th }}$ cluster at position $x_{n}>x_{n-1}$ and no other cluster in the gas gap is given by

$$
P\left(x_{1}\right) P\left(x_{2}-x_{1}\right) \ldots P\left(x_{n}-x_{n-1}\right)\left(1-\int_{0}^{d-x_{n}} P(y) d y\right)=\frac{1}{\lambda^{n}} e^{-\frac{d}{\lambda}}
$$

The probability to have exactly $n$ electrons in the gas gap, independent of position, is given by the integral over all positions

$$
\int_{0}^{d} \int_{0}^{x_{n}} \int_{0}^{x_{n-1}} \cdots \int_{0}^{x_{2}} \frac{1}{\lambda^{n}} e^{-\frac{d}{\lambda}} \mathrm{d} x_{1} \mathrm{~d} x_{2} \ldots . \mathrm{d} x_{n}=\frac{1}{n !}\left(\frac{d}{\lambda}\right)^{n} e^{-\frac{d}{\lambda}}
$$

which is the expected Poisson distribution. The average number of avalanche electrons $\bar{N}^{-} \mathrm{n}$ given by

$$
\begin{aligned}
\bar{N}^{-} & =\sum_{n=1}^{\infty} \int_{0}^{d} \int_{0}^{x_{n}} \int_{0}^{x_{n-1}} \ldots \int_{0}^{x_{2}} \frac{1}{\lambda^{n}} e^{-\frac{d}{\lambda}} n_{a v}\left(\bar{n}\left(d-x_{1}\right)+\bar{n}\left(d-x_{2}\right)+\ldots+\bar{n}\left(d-x_{n}\right)\right) \mathrm{d} x_{1} \mathrm{~d} x_{2} \ldots . \mathrm{d} x_{n} \\
& =\sum_{n=1}^{\infty} \frac{n_{a v}\left(e^{(\alpha-\eta) d}-1\right) d^{n-1} e^{-\frac{d}{\lambda}}}{(n-1) !(\alpha-\eta) \lambda^{n}} \\
& =\frac{n_{a v}}{\lambda(\alpha-\eta)}\left(e^{(\alpha-\eta) d}-1\right) \\
& \approx \frac{n_{a v} e^{(\alpha-\eta) d}}{\lambda(\alpha-\eta)} \quad \text { for } \quad e^{(\alpha-\eta) d}>>1
\end{aligned}
$$

The average number of ions $\bar{N}^{+}$is derived by replacing $\bar{n}\left(d-x_{n}\right)$ by $\bar{p}\left(d-x_{n}\right)$ in the above expression and we find

$$
\bar{N}^{+}=\frac{n_{a v} \alpha}{\alpha-\eta}\left[\frac{1}{\lambda(\alpha-\eta)}\left(e^{(\alpha-\eta) d}-1\right)-\frac{d}{\lambda}\right] \approx \frac{n_{a v} \alpha}{\lambda(\alpha-\eta)^{2}} e^{(\alpha-\eta) d} \quad \text { for } \quad e^{(\alpha-\eta) d}>>1
$$


The average induced charge $\bar{Q}_{\text {ind }}$ is as before proportional to the number of ions

$$
\bar{Q}_{i n d}=\frac{E_{w}}{V_{w}} \frac{e_{0}}{\alpha} N^{+} \approx \frac{E_{w}}{V_{w}} \frac{n_{a v} e_{0}}{\lambda(\alpha-\eta)^{2}} e^{(\alpha-\eta) d} \quad \text { for } \quad e^{(\alpha-\eta) d}>>1
$$

The average RPC Signal is given by

$$
\begin{aligned}
& \bar{i}(t)=\sum_{n=1}^{\infty} \int_{0}^{d} \int_{0}^{x_{n}} \int_{0}^{x_{n-1}} \ldots \int_{0}^{x_{2}} \frac{1}{\lambda^{n}} e^{-\frac{d}{\lambda}}\left(i\left(t, x_{1}\right)+i\left(t, x_{2}\right)+\ldots+i\left(t, x_{n}\right)\right) d x_{1} d x_{2} \ldots d x_{n} \\
& =\sum_{n=1}^{\infty} \int_{0}^{d} \int_{0}^{x_{n}} \int_{0}^{x_{n-1}} \ldots \int_{0}^{x_{2}} \frac{1}{\lambda^{n}} e^{-\frac{d}{\lambda}} \frac{E_{w}}{V_{w}} n_{a v} e_{0} v e^{(\alpha-\eta) t}\left(\sum_{k=1}^{n} \Theta\left(\frac{d-x_{n}}{v}-t\right)\right) d x_{1} d x_{2} \ldots . d x_{n} \\
& =\sum_{n=1}^{\infty} \frac{E_{w}}{V_{w}} n_{a v} e_{0} v e^{(\alpha-\eta) t} \frac{1}{\lambda} e^{-\frac{d}{\lambda}}(d-v t) \Theta\left(\frac{d}{v}-t\right)\left(\frac{d}{\lambda}\right)^{n-1} \frac{1}{(n-1) !} \\
& =\frac{E_{w}}{V_{w}} n_{a v} e_{0} v e^{(\alpha-\eta) t} \frac{1}{\lambda}(d-v t) \Theta\left(\frac{d}{v}-t\right)
\end{aligned}
$$

We find that the average RPC signal is equal to the enveloping function from Eq. 23. 


\section{$7 \quad$ Intrinsic Timing}

In this section we want to find an order of magnitude formula for the intrinsic timing of a single gap RPC. The timing should not be affected by saturation effects since at the threshold level space charge effects are not yet important. We assume a single primary electron somewhere in the RPC. The RPC signal and amplitude fluctuation is then given by Eq. 21. Setting a threshold of $A_{t h r}$ to the RPC signal we find a threshold crossing time of

$$
i(t)=A e^{(\alpha-\eta) v t}=A_{t h r} \quad \rightarrow \quad t(A)=\frac{1}{(\alpha-\eta) v} \ln \frac{A_{t h r}}{A}
$$

The amplitude $A$ is exponentially distributed around some average amplitude $A_{a v}$. Therefore the time distribution $P(t)$ for a given threshold is given by

$$
\begin{aligned}
P(t) & =\int_{0}^{\infty} \frac{1}{A_{a v}} e^{-\frac{A}{A_{a v}}} \delta\left(t-\frac{1}{(\alpha-\eta) v} \ln \left(\frac{A_{t h r}}{A}\right)\right) \mathrm{d} A \\
& =\frac{(\alpha-\eta) v A_{t h r}}{A_{a v}} \exp \left(-\frac{(\alpha-\eta) v t}{A_{a v}}-\frac{A_{t h r}}{A_{a v}} e^{-(\alpha-\eta) v t}\right)
\end{aligned}
$$

where $\delta(x)$ is the Dirac delta function. This distribution has the curious property that a different threshold merely corresponds to a time shift, i.e. the shape of the distribution is independent of threshold and average amplitude. Time shifting the maximum to zero, the distribution reads

$$
P(t)=(\alpha-\eta) v F((\alpha-\eta) v t) \quad F(x)=\exp (-x-\exp (-x))
$$

The function is shown in Fig. 10. The variance $\sigma$ of the function $F(x)$ is given by

$$
\sigma(F)=1.28
$$

so the RPC time resolution is given by

$$
\sigma_{t}=\frac{1.28}{(\alpha-\eta) v}
$$

where $\alpha-\eta$ is the effective Townsend coefficient and $v$ is the electron drift velocity. We therefore expect that the intrinsic time resolution depends only on the drift velocity and the effective Townsend coefficient and not on the threshold. This is reproduced by the detailed Monte Carlo simulation (Fig. 10b) and also observed in measurements [10].

For the timing RPC with parameters from Eq. $24 \sigma_{t} \approx 50 \mathrm{ps}$ and for Trigger RPCs with parameters from Eq. 25 we find $\sigma_{t} \approx 1 \mathrm{~ns}$. These numbers are quite close to the ones quoted in [24] and [25]. 


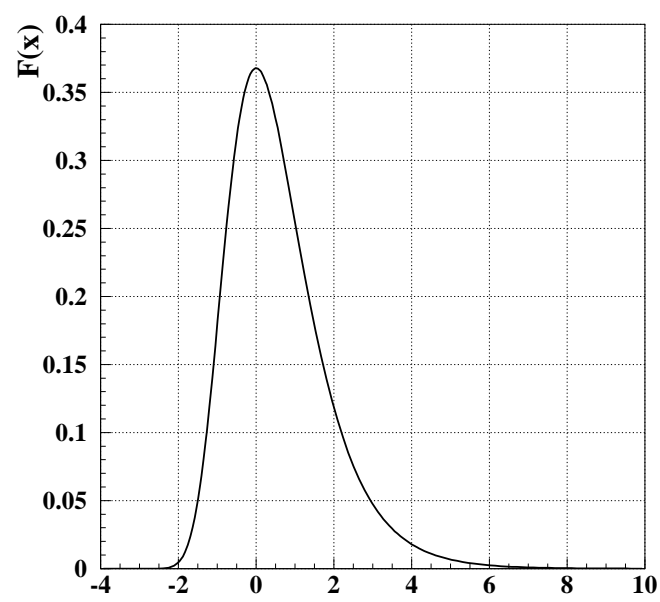

a)

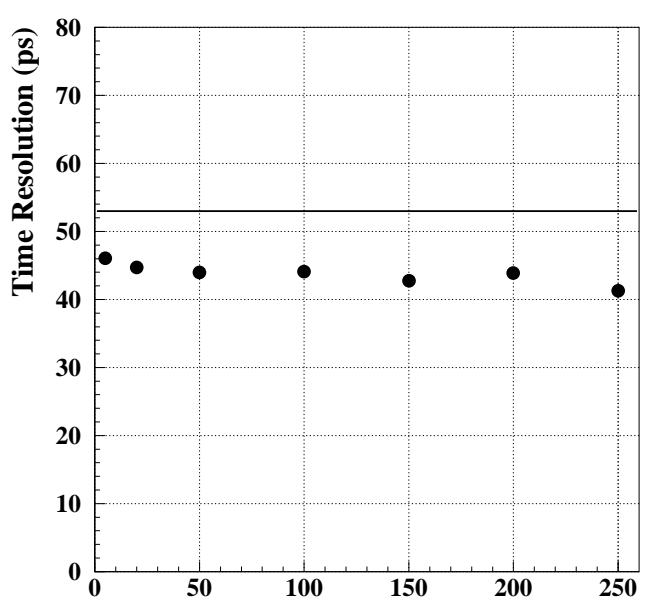

Discriminator Threshold (fC)

b)

Figure 10: (a) The function $F(x)$ from Eq. 44 giving the RPC time resolution. The time resolution scales with $1 /(\alpha-\eta) v$ which just 'stretches the abscissa'. (b) Full Monte Carlo simulation of the time resolution versus threshold for the $300 \mu \mathrm{m} \mathrm{RPC} \mathrm{at} 3 \mathrm{kV}$ with $t_{p}=0.5 \mathrm{~ns}$ and ENC $1 \mathrm{fC}$. The solid line shows Eq. 46. 
Efficiency

In this section we want to estimate the efficiencies that we expect with the detector physics model and numbers given before. In a simplified view we expect the RPC to be efficient if the first cluster creates an avalanche that exceeds the threshold or the first cluster is attached and the second cluster exceeds the threshold or the first and second cluster are attached and the third exceeds the threshold etc. In addition we assume that the clusters contain only one electron and we neglect avalanche fluctuations, i.e. a primary electron at position $x$ in the gas gap will induce a charge of

$$
Q_{\text {ind }}(x)=\frac{E_{w}}{V_{w}} \frac{e_{0}}{\alpha-\eta} e^{(\alpha-\eta)(d-x)}-1
$$

on the readout electrode. Setting a threshold of $Q_{t}$, the condition for an efficient event is $Q_{\text {ind }}(x)>Q_{t}$ meaning $x<x_{0}$ with

$$
x_{0}=d-\frac{1}{\alpha-\eta} \ln \left[1+\frac{V_{w}}{E_{w}} \frac{\alpha-\eta}{e_{0}} Q_{t}\right]
$$

The probability that the first cluster is not attached and above threshold is

$$
P_{1}=\left(1-\frac{\eta}{\alpha}\right) \int_{0}^{x_{0}} \frac{1}{\lambda} e^{-\frac{x}{\lambda}} \mathrm{d} x
$$

The probability that the first cluster is attached and the second one is not attached and above threshold is

$$
P_{2}=\int_{0}^{x_{0}} \int_{0}^{x_{2}} \frac{\eta}{\alpha} \frac{1}{\lambda} e^{-\frac{x_{1}}{\lambda}}\left(1-\frac{\eta}{\alpha}\right) \frac{1}{\lambda} e^{-\frac{x_{2}-x_{1}}{\lambda}} \mathrm{d} x_{1} \mathrm{~d} x_{2}
$$

Continuing the series and evaluating the integrals, the probability for the $n^{\text {th }}$ cluster to be above threshold and the $n-1$ before to be attached is

$$
P_{n}=\left(\frac{\eta}{\alpha}\right)^{n-1}\left(1-\frac{\eta}{\alpha}\right)\left(1-K\left[\frac{x_{0}}{\lambda}, n\right]\right)
$$

where $K[x, n]$ is from Eq. 26. The efficiency $\varepsilon$ is then given by

$$
\varepsilon=\sum_{n=1}^{\infty} P_{n}=1-e^{-\left(1-\frac{\eta}{\alpha}\right) \frac{d}{\lambda}}\left[1+\frac{V_{w}}{E_{w}} \frac{\alpha-\eta}{e_{0}} Q_{t}\right]^{\frac{1}{\alpha \lambda}}
$$

The efficiency depends explicitly on $\alpha$ and $\eta$ and not just on the effective Townsend coefficient. For $\alpha \rightarrow \infty$ the inefficiency is $\exp [-d / \lambda]$ which is the probability that there is no primary cluster in the gas gap. This formula together with a full Monte Carlo simulation is shown in Fig. 11. For the $2 \mathrm{~mm}$ RPC the formula underestimates the efficiency since it does not take into account the possibilities that individual avalanches stay below the threshold but that the sum crosses the threshold. However, the order of magnitude of the efficiency can be estimated quite well. 


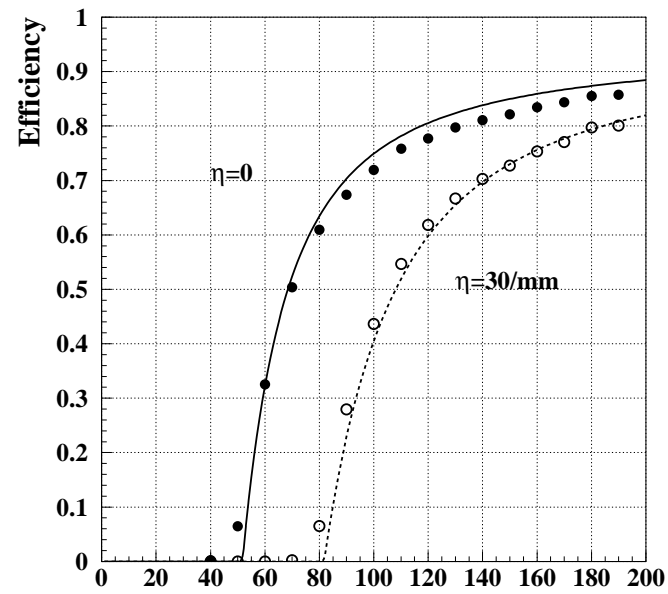

a)

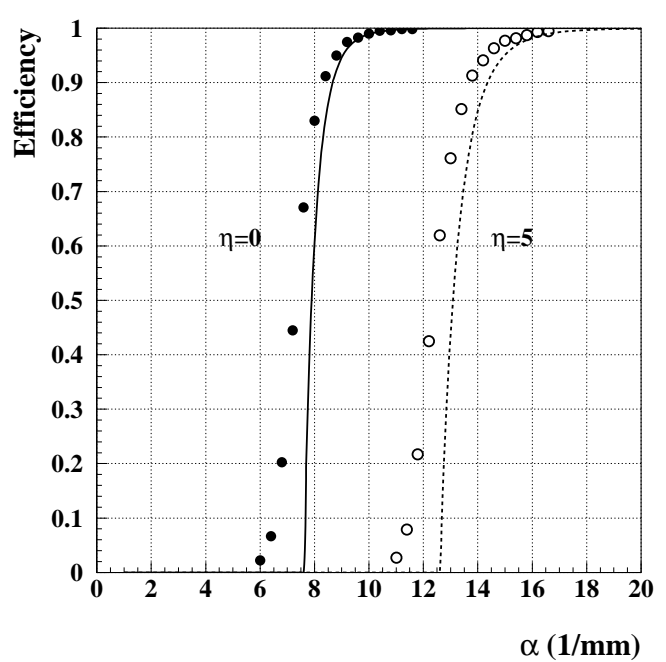

b)

Figure 11: Efficiency from Eq. 52 together with the full Monte Carlo for a $0.3 \mathrm{~mm}$ RPC (a) and a $2 \mathrm{~mm}$ RPC (b). 


\section{9}

\section{Space Charge Effects}

Inserting the detector physics parameters from Eq. 24 and 25 in Eq. 38 and 39 we find average charges that are significantly larger than the measured ones (measurements in brackets)

$$
\begin{array}{lll}
\text { Timing RPC: } & Q_{t o t} \approx 1.7 \times 10^{7}(5) \mathrm{pC} & Q_{\text {ind }} \approx 1.4 \times 10^{5}(0.5) \mathrm{pC} \\
\text { Trigger RPC: } & Q_{t o t} \approx 3300(40) \mathrm{pC} & Q_{\text {ind }} \approx 100(2) \mathrm{pC}
\end{array}
$$

The discrepancy for the total charge value is a factor $\approx 3.4 \times 10^{6}$ for the timing RPCs and $\approx 10^{2}$ for the trigger RPCs. Using Eq. 37 we find the average number of avalanche electrons for the timing RPC to be $\approx 10^{14}$. Assuming a single electron avalanche in the timing RPC, the electron cloud will assume a Gaussian shape with $\sigma \approx 20 \mu \mathrm{m}$ after $300 \mu \mathrm{m}$ due to longitudinal and transverse diffusion. Assuming a sphere of charge with $10^{6}$ electrons and radius of $20 \mu \mathrm{m}$, the field on the surface is $36 \mathrm{kV} / \mathrm{cm}$, so for numbers of $10^{6}-10^{7}$ electrons, the fields in the avalanche become comparable to the applied field. Therefore space charge effects must play a significant role in this detector. It is shown in [21] that taking into account the field of the avalanche correctly explains the observed charges.

In this report we are mainly interested in timing and efficiency of RPCs which are not influenced by space charge. At the typical RPC thresholds of 10-100 fC the avalanches are still small and not affected by space charge. We will take the effect into account in a crude way by allowing the avalanche growth only up to a certain size as proposed in [4].

\section{Avalanche Statistics at High Fields}

The assumption that the ionisation probability is independent of the history of previous collisions will not hold above a certain electric field value. Considering a Townsend coefficient of $\alpha=123 / \mathrm{mm}$ at the electric field $E=100 \mathrm{kV} / \mathrm{cm}$, the average distance between ionising collisions $1 / \alpha$ is $8.13 \mu \mathrm{m}$. Assuming an ionisation energy of $U_{i}=25 \mathrm{eV}$ an electron has to travel a distance of $x_{0}=U_{i} / E=2.5 \mu \mathrm{m}$ after a collision to again reach this energy, so within $2.5 \mu \mathrm{m}$ after each collision the ionisation probability is zero. Since this number is comparable to $1 / \alpha$ the condition that the ionisation probability is independent of the previous collisions does not hold any more and the avalanche fluctuations will be altered. Instead the shape of the distribution depends on the parameter [22]

$$
r=\frac{1}{\alpha} \frac{E}{U_{i}}
$$

At low fields $(r<<1)$ the avalanches started by a single electron (and multiplying over a fixed distance) result in the exponential distribution described in the previous chapter. At high fields $(r \approx 1)$ the distributions show a pronounced maximum for which many different interpretations were given [23]. A popular way to describe this phenomenon is the Polya distribution which derived from the probability $p$ to find $n+1$ electrons in $x+\mathrm{d} x$ as

$$
p=n \lambda\left(b-\frac{1-b}{n}\right) \mathrm{d} x
$$

We see that this distribution assumes that the probability to create an electron depends on the current size of the avalanche. This however misses a clear physical interpretation and describes some kind of space charge effect which we include in the way mentioned above. Therefore the only justification for this distribution is that it can parametrise the measured curves in a nice way. 
For this study we will as in sect. 4 follow a model by Legler [19] which assumes the physical picture mentioned above. If $\xi$ is the distance travelled by an electron from the last ionising collision the ionisation probability will be given by $a(\xi) \mathrm{d} x$ where $a(\xi)$ is zero for $\xi<x_{0}$ and will increase for $\xi>x_{0}$. In the same manner the attachment coefficient will depend on $\xi$ and we replace the constant attachment coefficient $\eta$ by $e(\xi)$. Starting with a single electron at $x=0$, the average number of avalanche electrons at a distance $x$ that had the last ionising collision at a distance between $\xi$ and $\xi+\mathrm{d} \xi$ from $x$ is given by [19]

$$
n(x, \xi)=A e^{\lambda x} e^{-\lambda \xi-\int_{0}^{\xi}\left[a\left(\xi^{\prime}\right)+e\left(\xi^{\prime}\right)\right] \mathrm{d} \xi^{\prime}}
$$

with boundary condition

$$
n(x, 0)=2 \int_{0}^{\infty} a(\xi) n(x, \xi) \mathrm{d} \xi
$$

The parameter $\lambda$ is defined by the boundary condition and $A$ is a normalization constant. This equation is the pendant to Eq. 3 . We see that the average number of electrons increases exponentially for any given function $a(\xi)$ and $e(\xi)$.

The equation determining the statistical fluctuation for this model is difficult to solve and we just show Monte Carlo results for different parameters. As a simple model we assume the function $a(\xi)$ to be zero for $\xi<x_{0}$ and $a(\xi)=a_{0}$ for $\xi>x_{0}$ and assume $e(\xi)=\eta$ to be constant. Fig. 12 shows an example for a single electron avalanche spectrum for $\alpha=123 / \mathrm{mm}, x_{0}=0,2,4 \mu \mathrm{m}$. For large $x_{0}$ values the charge spectrum shows a pronounced peak.

This avalanche statistics effect has however a very small influence on the charge spectrum of the $0.3 \mathrm{~mm}$ and $2 \mathrm{~mm}$ RPCs since the position fluctuations of the primary electrons completely dominate the avalanche fluctuation as shown in the next section.

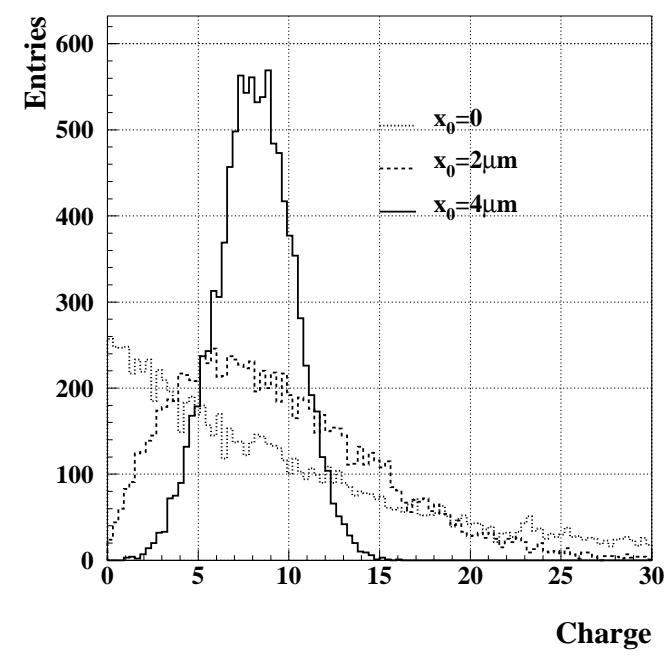

Figure 12: Charge distributions for avalanches started by a single electron and multiplying over a fixed distance of $300 \mu \mathrm{m}$ for $\alpha=123 / \mathrm{mm}, x_{0}=0,2,4 \mu \mathrm{m} .1 / \alpha=8.13 \mu \mathrm{m}$. For $x_{0}$ values approaching $1 / \alpha$ the the charge spectrum shows a pronounced peak. 


\section{Monte Carlo and Comparison with Experiment}

Finally we want to compare the simulation procedure and detector physics parameters, outlined in the previous sections, to experimental results. The simulation procedure for a single event is the following

- The gas gap of size $d$ is divided into $N_{\text {step }}$ steps of size $\Delta x=d / N_{\text {step }}$ corresponding to time steps of $\Delta t=\Delta x / v$ where $v$ is the electron drift velocity from Fig. 7 at the given field.

- Primary clusters are distributed along the gas gap at distances following an exponential distribution with the mean taken from Fig. 3a.

- Primary electrons are put to each cluster following the cluster size distribution from Fig. 3b.

- The avalanche for each single electron is simulated using Eq. 15, 16, 17 and the procedure outlined in section 4 with numbers for Townsend and attachment coefficient from Fig. 4. This provides $N(t)$, the number of electrons at time $t$.

- If $N(t)$ exceeds a specified value $N_{\text {sat }}$ the avalanche growth is stopped and the $N_{\text {sat }}$ electrons propagate to the gap end. This procedure simulates the space charge effect.

- The induced current signal is then calculated with Eq. 19 where the electron drift velocity is from Fig. 7 and the weighting field is from Eq. 20.

- In each simulation step the electrons are propagated by $\Delta x(\Delta t)$, the electrons leaving the gas gap are subtracted from $N(t)$, so the total signal has a maximum duration of $T \leq d / v$. We include the electronics by convoluting the RPC signal with the amplifier delta response $f(t)$

$$
h(s)=\frac{n^{-n} e^{n} n ! \tau}{(1+s \tau)^{n+1}} \quad \rightarrow \quad f(t)=\mathcal{L}^{-1}[h(s)]=n^{-n} e^{n}\left(\frac{t}{\tau}\right)^{n} e^{-\frac{t}{\tau}}
$$

where $t_{p}=n \tau$ is the peaking time and $n$ corresponds to the number of amplifier integration stages. The noise is included by adding Gaussian numbers to the signal in each time bin with a $\sigma$ giving the correct Equivalent Noise Charge (ENC) at the output.

\subsection{Timing RPCs}

Fig. 13a shows a simulated charge spectrum for geometry from Fig. $1 \mathrm{c}$ at $3 \mathrm{kV}(\mathrm{E}=100 \mathrm{kV} / \mathrm{cm})$. First of all, the shape of the spectrum and the $25 \%$ inefficiency match quite well the numbers reported in [24] and [11]. Overlayed is a simulation taking into account the high field avalanche statistics effect from the previous section. Although the charge spectrum for an electron multiplying over a fixed distance is strongly affected by the value of $x_{0}$ (Fig. 12), the RPC spectrum shows no effect whatsoever, which is due to the fact that the charge fluctuations due to the primary ionization positions are much larger. The charge spectra for three different voltages for the quad-gap RPC from Fig. 1b are shown in Fig. 13b. The spectra are equal to the 4 times self convoluted charge spectrum from the single gap RPC and resemble quite well the ones presented in [10]. Fig. 14 shows efficiency and time resolution versus voltage for single and quad gap RPC. The single gap RPC was simulated for the geometry from Fig. 1c giving a weighting field of $1.25 / \mathrm{mm}$ with $7 \mathrm{GeV}$ pions i.e. 9.4 clusters $/ \mathrm{mm}, 20 \mathrm{fC}$ threshold, $500 \mathrm{ps}$ amplifier peaking time, $1 \mathrm{fC}$ noise and Townsend coefficient, attachment coefficient and drift velocity for the gas $\mathrm{C}_{2} \mathrm{~F}_{4} \mathrm{H}_{2} / \mathrm{i}-\mathrm{C}_{4} \mathrm{H}_{10} / \mathrm{SF}_{6} 85 / 5 / 10$. The overlayed data are from [24].

The simulation for the quad gap RPC was done with the same parameters, except for the weighting field b) with $1.026 / \mathrm{mm}$ and an amplifier peaking time of $3 \mathrm{~ns}$. The overlayed data are from [9]. Fig. 15 shows the charge-time correlation for the quad gap RPC.The agreement between measurements and simulation is quite acceptable. 

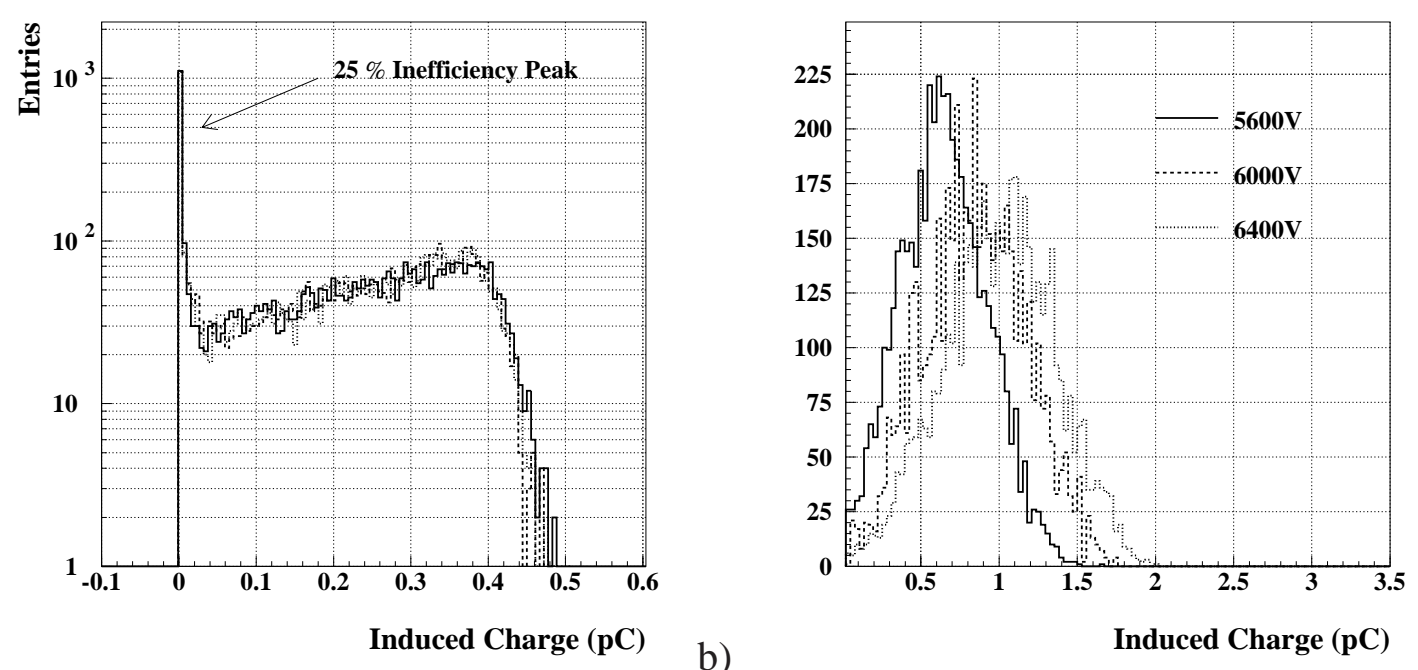

Figure 13: a) Charge spectra for the single gap timing RPC assuming saturation at $N_{\text {sat }}=$ $1.6 \times 10^{7}$ electrons. The inefficiency is $25 \%$ for a $20 \mathrm{fC}$ threshold. Three histograms for $x_{0}=$ $0, x_{0}=2.5 \mu \mathrm{m}, x_{0}=5 \mu \mathrm{m}$ are overlayed and show essentially no difference. $\mathrm{b}$ ) Charge spectra for the quad gap timing RPC assuming Townsend and attachment coefficients from Fig. 4. The avalanche in each gap is saturated at $N_{\text {sat }}=1.6 \times 10^{7}$ electrons.
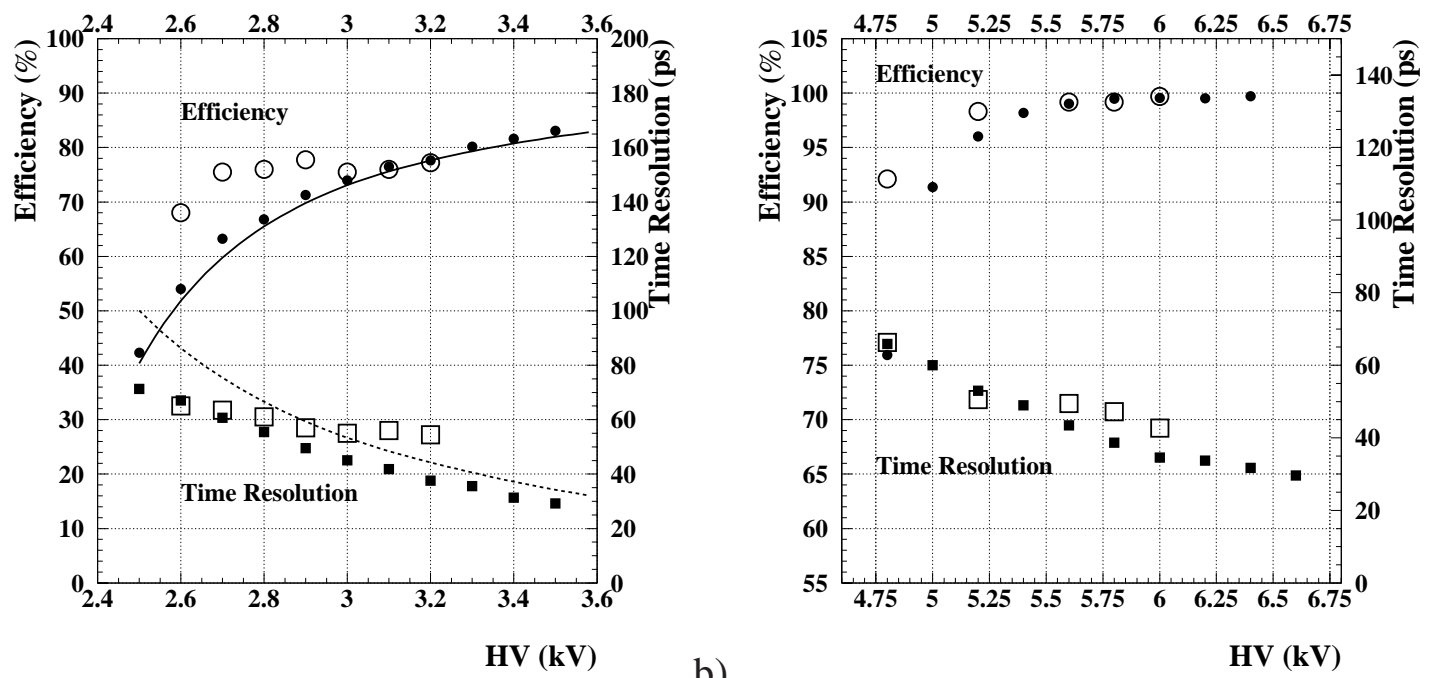

a)

b)

Figure 14: Results for efficiency and amplitude corrected time resolution for the single gap (a) and quad gap RPC (b) for the parameters mentioned in the text. The open symbols are measurements. For the single gap RPC the formulas for time resolution and efficiency from Eq. 46 and 52 are overlayed. 


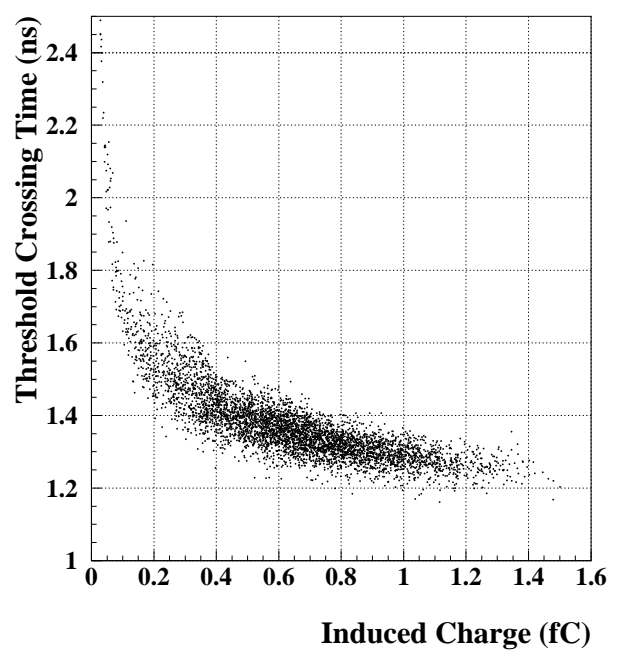

Figure 15: Charge to time correlation for the quad gap RPC at $5600 \mathrm{~V}$. 


\subsection{Trigger RPCs}

Fig. 16a shows simulated efficiency and time resolution for the RPC from Fig. 2 together with measurements from [25]. The efficiency plateau is shifted by about $500 \mathrm{~V}$. Multiplying the attachment coefficient by 0.45 the simulated and experimental curves match. The simulated time resolution is better than the $1.1 \mathrm{~ns}$ quoted in [25]. Fig. 16b) shows the charge spectra for the Trigger RPC for different operating voltages with $\eta$ scaled by 0.45 . The spectra are again unaffected by realistic $x_{0}$ parameters. The simulated spectra show a sharper cutoff at high charges than the measured ones which is due to the crude space charge effect approximation.

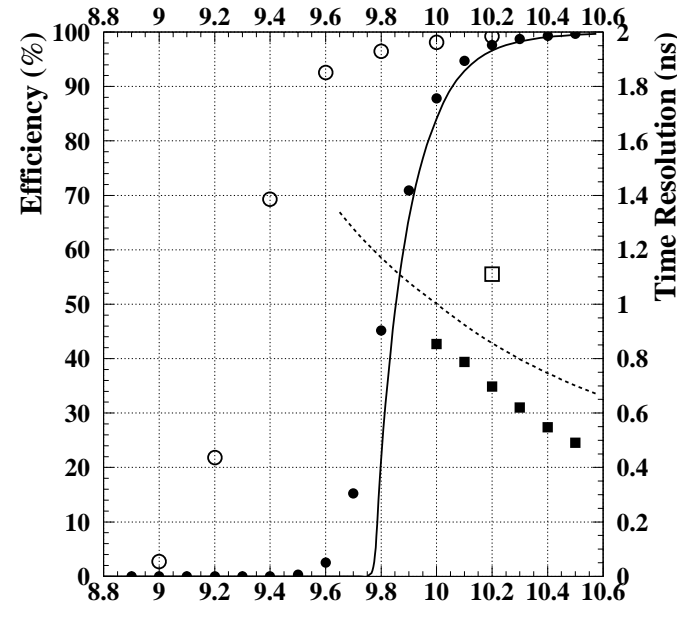

a)
HV (kV)

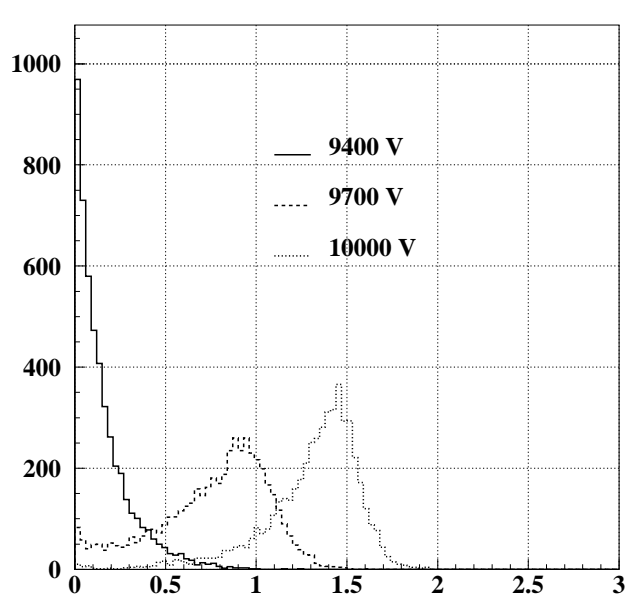

b)

Induced Charge (pC)

Figure 16: (a) Simulated efficiency (black circles) and time resolution (black squares) for trigger RPCs together with measurements (open symbols) from [25]. The curves are shifted by about $500 \mathrm{~V}$. Scaling the attachment coefficient with 0.45 matches simulation and experiment. The simulated time resolution (back squares) is significantly better that the measured one. (b) Charge spectra of the trigger RPC for saturation at $N_{\text {sat }}=2.5 \times 10^{7}$ electrons, and scaled attachment coefficient. 


\section{Effect of Number of Gaps}

In this section we want to investigate the effect of different numbers of gaps on time resolution and efficiency. The weighting fields for an RPC with $n$ gas gaps of size $d$ separated by $n-1$ glass plates of thickness $b$ and permittivity $\varepsilon$ is

$$
\frac{E_{w}}{V_{w}}=\frac{\varepsilon}{n d \varepsilon+(n-1) b} \quad n>1
$$

For the single gap RPC $(n=1)$ we use the geometry from Fig. 1a with corresponding weighting field from Eq. 20a. Since the weighting field decreases with the number of gaps the total induced charge is almost independent of the gap number. We assume an applied voltage that gives a field of $100 \mathrm{kV} / \mathrm{cm}$ in the gas gaps i.e. $3 \mathrm{kV}$ for single gap RPC, $6 \mathrm{kV}$ for double gap RPC etc. Fig. 17 shows the time resolution and efficiency versus gap number. The Figure also shows the $1 / \sqrt{n}$ scaling of the single gap time resolution and $1-(1-\varepsilon)^{n}$ scaling of the single gap efficiency. We see that the efficiency follows the simple scaling considerations, the time resolution improvement however is less than one expects from naive scaling. The reason is that the timing is dominated by the gap with the largest signal. The largest signal gives the earliest threshold crossing time, so the timing of the multi gap RPC is approximately given by the 'earliest gap'. The earliest of $n$ time measurements however has a larger r.m.s. than the average of $n$ time measurements.

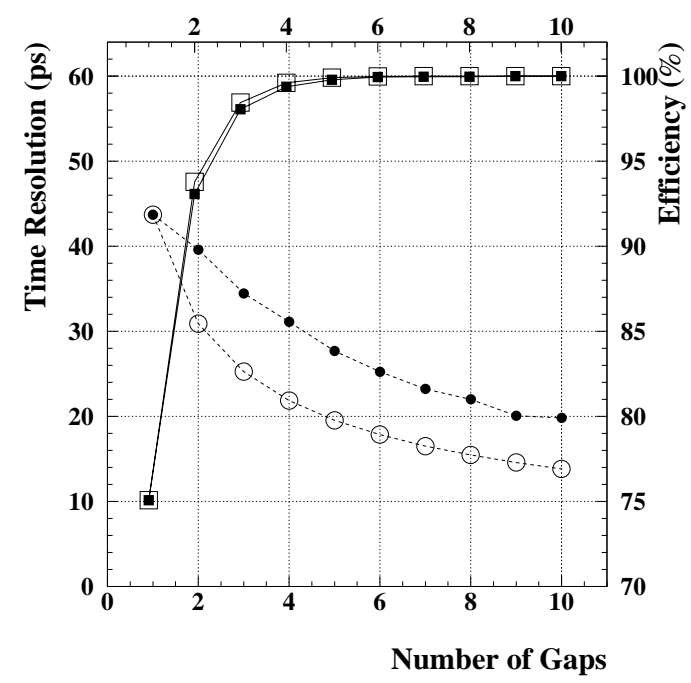

Figure 17: Efficiency (black squares) and amplitude corrected time resolution (black circles) versus number of gas gaps. The open symbols give the numbers that are expected from naive scaling. 


\section{Amplifier Bandwidth and Noise}

In this section we study the dependence of the RPC time resolution on the amplifier bandwidth. We characterise the amplifier by it's peaking time $t_{p}$ and order $n$ as given in Eq. 58 . We use the $300 \mu \mathrm{m}$ single gap timing RPC at $3 \mathrm{kV}$ as an example. Fig. 18 shows the time resolution versus amplifier peaking time. Neglecting the noise, the time resolution is independent of peaking time since using the charge-time correlation one can fully correct for the introduced time slewing effects. Including the noise however shows that for slow amplifiers the intrinsic time resolution cannot be recovered. The reason is that the time jitter due to the noise (which cannot be corrected) inceases for slower signal rise time.

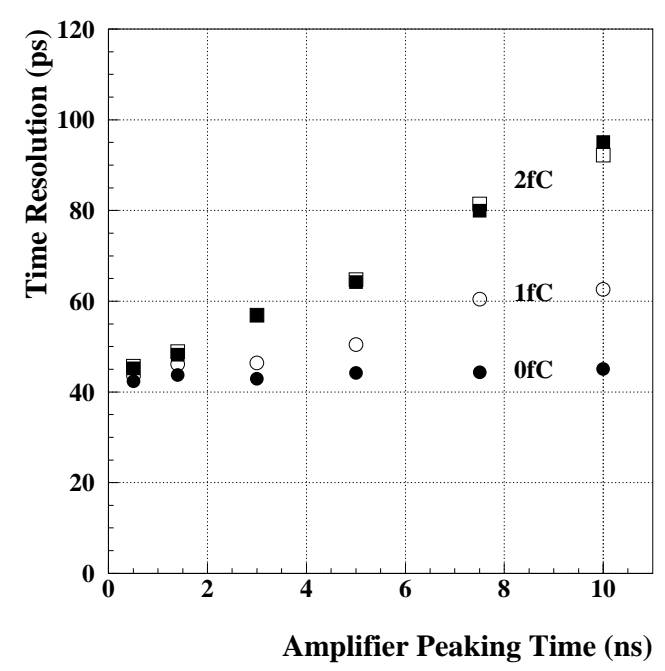

Figure 18: Simulation of the amplitude corrected time resolution versus amplifier peaking time for the $300 \mu \mathrm{m}$ single gap RPC with a threshold of $20 \mathrm{fC}$ and Equivalent Noise Charge of $0,1,2 \mathrm{fC}$. Electronics noise introduces a jitter that can not be recuperated by performing the amplitude correction. The influence of the noise therefore increases with the amplifier peaking time. For the $2 \mathrm{fC}$ curve the results for two different noise spectra are shown. 


\section{Conclusions}

We have presented an RPC simulation procedure including all detector physics and electronics effects from primary ionisation up to the frontend electronics output. Assuming a very prominent space charge effect that is modelled by simply stopping the avalanche growth at a certain number of electrons, we can reproduce the observed RPC performance numbers quite well without any additional assumptions. We only assume physical parameters as given by Heed [6], Magboltz [7] and Imonte [8]. The outlined simulation procedure can be implemented in a Monte Carlo program in a very simple way. Generally we can conclude that

- Neglecting space charge effects, the calculated average avalanche charges for the $300 \mu \mathrm{m}$ timing RPCs are a factor $10^{7}$ larger than the measured ones. This shows that space charge effect play a significant role in RPCs [21].

- The RPC efficiency is approximately given by $1-e^{-\left(1-\frac{\eta}{\alpha}\right) \frac{d}{\lambda}}\left[1+\frac{V_{w}}{E_{w}} \frac{\alpha-\eta}{e_{0}} Q_{t}\right]^{\frac{1}{\alpha \lambda}}$. It depends explicitly on the attachment coefficient and not just on the effective Townsend coefficient.

- The RPC time resolution is approximately given by $\sigma_{t}=1.28 /(\alpha-\eta) v$ and is independent of the applied threshold.

- The high efficiency (75\%) of single gap RPCs with $300 \mu \mathrm{m}$ gas gap is explained by the large primary ionisation density $(9.4 / \mathrm{mm})$ of the tetrafluorethane gas together with a very large effective Townsend coefficient of about 115/mm.

- Secondary particles produced in the RPC material should not play an important role in the RPC behaviour.

- The specific avalanche fluctuation model does not have a very large impact on the RPC charge spectrum for the RPCs discussed in this report, since in the given operating conditions the charge fluctuations due to primary ionisation position fluctuations are dominating.

- The time resolution for an $n$ gap RPC does not scale with $\sigma_{t} / \sqrt{n}$ where $\sigma_{t}$ is the single gap RPC time resolution. The efficiency however does scale with the expected scaling law of $1-(1-\varepsilon)^{n}$ where $\varepsilon$ is the efficiency of the single gap RPC.

- Neglecting electronics noise, the amplifier bandwidth has very little influence on the time resolution since the time slewing introduced by slow amplifiers can be fully corrected by the charge-time correlation. Electronics noise however introduces a jitter at the threshold level which has more effect for slow amplifiers and the intrinsic time resolution can not be recuperated.

We want to thank Paolo Fonte for many interesting discussions. 


\section{References}

[1] W. Riegler, Induced signals in resistive plate chambers, CERN-EP-2002-024, march 2002, to be published in NIMA.

[2] W. Riegler, D. Burgarth, Signal propagation, termination, crosstalk and losses in resistive plate chamber, NIM A481(2001) 130-143

[3] M. Abbrescia et al., Resistive plate chambers in avalanche mode : a comparison between model predictions and experimental results, NIMA 409 (1998).

[4] M. Abbrescia et al., Progress in the simulation of Resistive Plate Chambers in avalanche mode, CMS conference report CMS CR 1998/021.

[5] P. Fonte, High-resolution timing of MIPs with RPCs - a model, NIMA 456 (2000) 6-10

[6] Igor Smirnov, Heed, program to compute energy loss of fast particles in gases, Version 1.01, CERN

[7] S. Biagi, Magboltz, program to compute gas transport parameters, Version 2.2, CERN

[8] S. Biagi, Imonte, program to compute gas properties, Version 4.5

[9] P. Fonte et al., High-resolution RPCs for large TOF systems, NIM A449 (2000) 295-301

[10] A. Akindinov, P. Fonte et al., A four-gap glass-RPC time-of-flight array with $90 \mathrm{ps}$ time resolution, CERN-EP 99-166, October 1999

[11] P. Fonte, V. Peskov, High-Resolution TOF with RPCs, preprint LIP/00-04, October 2000

[12] Addendum to the Technical Design Report of the Time of Flight System (TOF), ALICE Collaboration, ALICE-TDR-8-ADD-1, CERN-LHCC-2002-016, CERN, 24 April 2002.

[13] R. Santonico, R. Cardarelli, Development of Resistive Plate Counters, NIM A263 (1981) et al.

[14] ATLAS Muon Spectrometer Technical Design Report, CERN-LHCC-97-22, ATLAS TDR 10, CERN 1997.

[15] R. Camarri et al., Streamer suppression with SF6 in RPCs operated in avalanche mode, NIM A414 (1998) 317-324

[16] F. Rieke and W. Prepejchal, Ionization Cross Sections of Gaseous Atoms and Molecules for High-Energy Electrons and Positrons, Phys. Rev. A 6, 1507 (1972)

[17] A. Fasso et al., FLUKA 99, Particle Transport Code, Version 1999.

[18] Helmut Vincke, CERN, private communication

[19] Werner Legler, Die Statistik der Elektronenlawinen in elektronegativen Gasen, bei hohen Feldstärken und bei grosser Gasverstärkung, Z. Naturforschung. 16a, 253-261 (1961)

[20] S. Ramo, Currents induced in electron motion, PROC. IRE 27, 584 (1939)

[21] C. Lippmann, W. Riegler, Space Charge Effects and Induced Signals in Resistive Plate Chambers, Presentation at the 6th Workshop on Resistive Plate Chambers and Related Detectors, Coimbra, Nov. 2001.

[22] Heinz Räther, Electron avalanches and breakdown in gases, Butterworth \& Co., 1964

[23] H. Genz, Single electron detection in proportional gas counters, NIM A112 (1973) 83-90

[24] P. Fonte, Development of Large Area and of Position Sensitive Timing RPCs, presentation at the 9th Vienna Conference on Instrumentation Vienna/Austria, February 19-23, 2001

[25] G. Aielli et al., Performance of a large-size RPC equipped with the final ATLAS front-end electronics at X5-GIF irradiation facility, NIMA 456(2000) 77-81. 